Mon. Not. R. Astron. Soc. 000, 田 (1998) Printed 14 August $2018 \quad$ (MN LATEX style file v1.4)

\title{
KPD 0422+5421: A New Short Period Subdwarf B/White Dwarf Binary
}

\author{
C. Koen ${ }^{1,2}$, Jerome A. Orosz ${ }^{3 \star} \&$ Richard A. Wade ${ }^{3}$ \\ ${ }^{1}$ South African Astronomical Observatory, P.O. Box 9, 7935 Observatory, South Africa \\ ${ }^{2}$ Department of Astronomy, University of Texas, Austin, TX 78712, USA \\ ${ }^{3}$ Department of Astronomy 83 Astrophysics, The Pennsylvania State University, 525 Davey Laboratory, University Park, PA 16802, USA
}

14 August 2018

\begin{abstract}
The sdB star KPD $0422+5421$ was discovered to be a single-lined spectroscopic binary with a period of $P=0.0901795 \pm\left(3 \times 10^{-7}\right)$ days (2 hours, 10 minutes). The $U$ and $B$ light curves display an ellipsoidal modulation with amplitudes of $\approx 0.02$ magnitudes. The sdB star contributes nearly all of the observed flux. This and the absence of any reflection effect suggest that the unseen companion star is small (i.e. $R_{\text {comp }} \approx$ $\left.0.01 R_{\odot}\right)$ and therefore degenerate. We modeled the $U$ and $B$ light curves and derived $i=78.05 \pm 0.50^{\circ}$ and a mass ratio of $q=M_{\text {comp }} / M_{\mathrm{sdB}}=0.87 \pm 0.15$. The sdB star fills $69 \%$ of its Roche lobe. These quantities may be combined with the mass function of the companion $\left(f(M)=0.126 \pm 0.028 M_{\odot}\right)$ to derive $M_{\mathrm{sdB}}=0.72 \pm 0.26 M_{\odot}$ and $M_{\text {comp }}=$ $0.62 \pm 0.18 M_{\odot}$. We used model spectra to derive the effective temperature, surface gravity, and helium abundance of the $\mathrm{sdB}$ star. We found $T_{\text {eff }}=25,000 \pm 1500 \mathrm{~K}$, $\log g=5.4 \pm 0.1$, and $[\mathrm{He} / \mathrm{H}]=-1.0$. With a period of 2 hours and 10 minutes, KPD $0422+5421$ has one of the shortest known orbital periods of a detached binary. This system is also one of only a few known binaries which contain a subdwarf $B$ star and a white dwarf. Thus KPD $0422+5421$ represents a relatively unobserved, and short-lived, stage of binary star evolution.
\end{abstract}

Key words: binaries: close — stars: variable — stars: individual (KPD 0422+5421)

\section{INTRODUCTION}

The star KPD 0422+5421 was discovered by Downes (1986) ' during the course of a search for very blue stars in the galactic plane, and classified as an sdB star, i.e. a hot, hydrogenrich subdwarf. R. Saffer (private communication) derived from spectroscopic measurements an effective temperature $T_{\text {eff }}=26,050$ and a surface gravity $\log g=5.51$; Saffer did not communicate a helium abundance. The sdB classification prompted one of the current authors to include KPD $0422+5421$ in a list of candidates to be monitored for rapid pulsations (see e.g. Kilkenny 1997a). During the course of the mandatory 90 minute high speed photometric run on the star, it appeared to vary with a period of about an hour, at an amplitude of roughly 0.01 mag. Further spectroscopic and photometric observations show that KPD $0422+5421$ is a close binary star with a period of 2.1643 hours, one of the shortest known orbital periods among the detached binaries. We argue that the companion star is a white dwarf, making KPD $0422+5421$ one of a small number of known

\footnotetext{
* Guest Observer, McDonald Observatory, University of Texas at Austin
}

systems with a sdB and a white dwarf. We report here our observations, data reductions, and data analysis.

\section{THE HIGH SPEED PHOTOMETRIC OBSERVATIONS AND ANALYSIS}

All the photometry reported here was obtained at the University of Texas' McDonald Observatory on Mt Locke, Texas. Two different instrumental configurations were used: the Louisiana State University Photometer (P-LSU) mounted on the $0.9 \mathrm{~m}$ telescope, and the Stiening Photometer attached to the $2.1 \mathrm{~m}$ telescope. The P-LSU is a standard two-channel photoelectric photometer (see e.g. Grauer \& Bond 1981), equipped with a blue-sensitive Hamamatsu R647 photomultiplier tube. The Stiening Photometer is a four-channel instrument which allows simultaneous high speed photometry in four different wavebands (Horne \& Stiening 1985; Wood, Zhang \& Robinson 1993). The current passbands of the instrument are similar to Johnson's $U B V R$, although there are notable differences (Wood et al. 1993). One channel of a second photometer (P45) was used as a fifth channel with the Stiening photometer, to monitor 
Table 1. Log of the observations, all of which were made at the University of Texas' McDonald Observatory. All $0.9 \mathrm{~m}$ observations were obtained under good photometric conditions, while all observations on the $2.1 \mathrm{~m}$ telescope were acquired under poor conditions. The instrumental configurations are described in the text. JD 2,450,752.8437 is 1997 October 31.3438 UT

\begin{tabular}{ccc}
\hline $\begin{array}{c}\text { Starting Time } \\
\text { JD 2,450,000+ }\end{array}$ & $\begin{array}{c}\text { Run Length } \\
\text { (Hours) }\end{array}$ & $\begin{array}{c}\text { Telescope } \\
\text { (meters) }\end{array}$ \\
\hline 752.8430 & 1.4 & 0.9 \\
753.8599 & 3.0 & 0.9 \\
754.7085 & 6.9 & 0.9 \\
785.7645 & 4.0 & 2.1 \\
787.7779 & 3.9 & 2.1 \\
\hline \hline
\end{tabular}

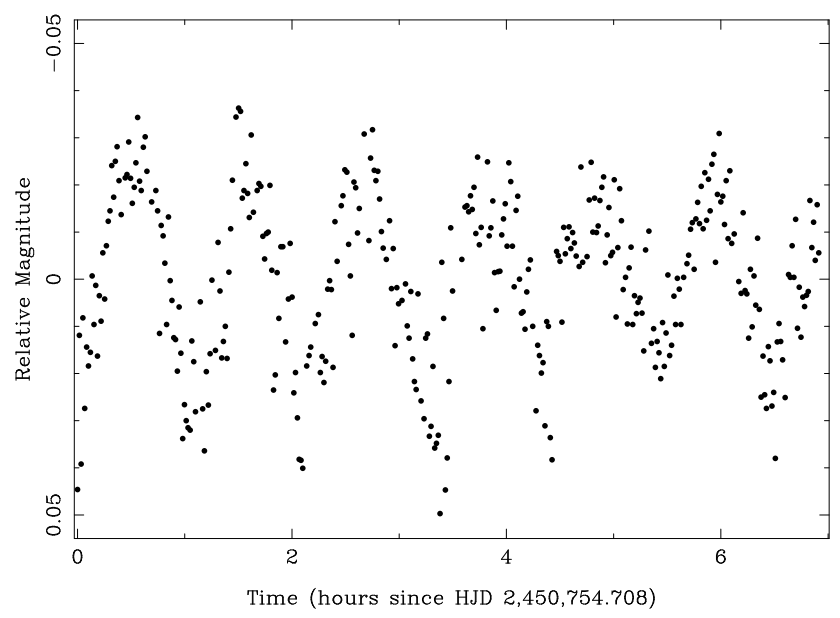

Figure 1. The white light observations obtained on JD 2,450,754 (1997 November 2 UT) using the $0.9 \mathrm{~m}$ telescope. One minute averages of the ten-second integrations are shown. The zeropoint has been set to the data mean value.

a comparison star. Observations with both P-LSU and P45 photometers were in "white light", i.e. no filters were placed in the light beam. The resulting effective wavelengths are similar to Johnson's $B$, but with substantially wider bandpasses. Integration of 10 and 5 seconds were used when running respectively the P-LSU and Stiening photometers.

The photometric runs are cataloged in Table 1; approximately 11 and 8 hours of data were obtained on the $0.9 \mathrm{~m}$ and $2.1 \mathrm{~m}$ telescopes respectively. The longest continuous light curve is plotted in Figure 1. There is a clear modulation with a period of $\approx 65$ minutes. Amplitude spectra of various combinations of photometric runs are shown in Figure 2. plotted over the frequency range of greatest interest. The low frequency content of the amplitude spectra has been removed to some extent by detrending the observations linearly. In addition, the fifth channel observations have been used to correct the data acquired on JD 2450785 for large atmospheric transparency drifts.

The one cycle per day aliasing pattern is clearly visible in the top panel of Figure a, which shows the amplitude spectrum of the combined $0.9 \mathrm{~m}$ data (from three successive nights). The aliasing pattern is much worse for the combined two $2.1 \mathrm{~m}$ runs (middle panel), since these were short. The bottom panel shows the amplitude spec-
Table 2. A listing of the largest peaks in the amplitude spectrum of all the high speed photometric data.

\begin{tabular}{ccc}
\hline $\begin{array}{c}\text { Frequency } \\
(\mathrm{c} / \mathrm{d})\end{array}$ & $\begin{array}{c}\text { Period } \\
\text { (days) }\end{array}$ & $\begin{array}{c}\text { Amplitude } \\
(\mathrm{mmag})\end{array}$ \\
\hline 22.055 & 0.045341 & 14.4 \\
22.086 & 0.045277 & 15.7 \\
22.117 & 0.045215 & 16.6 \\
22.148 & 0.045151 & 17.0 \\
22.178 & 0.045088 & 17.0 \\
22.209 & 0.045027 & 16.6 \\
22.240 & 0.044964 & 15.8 \\
22.271 & 0.044901 & 14.5 \\
\hline \hline
\end{tabular}

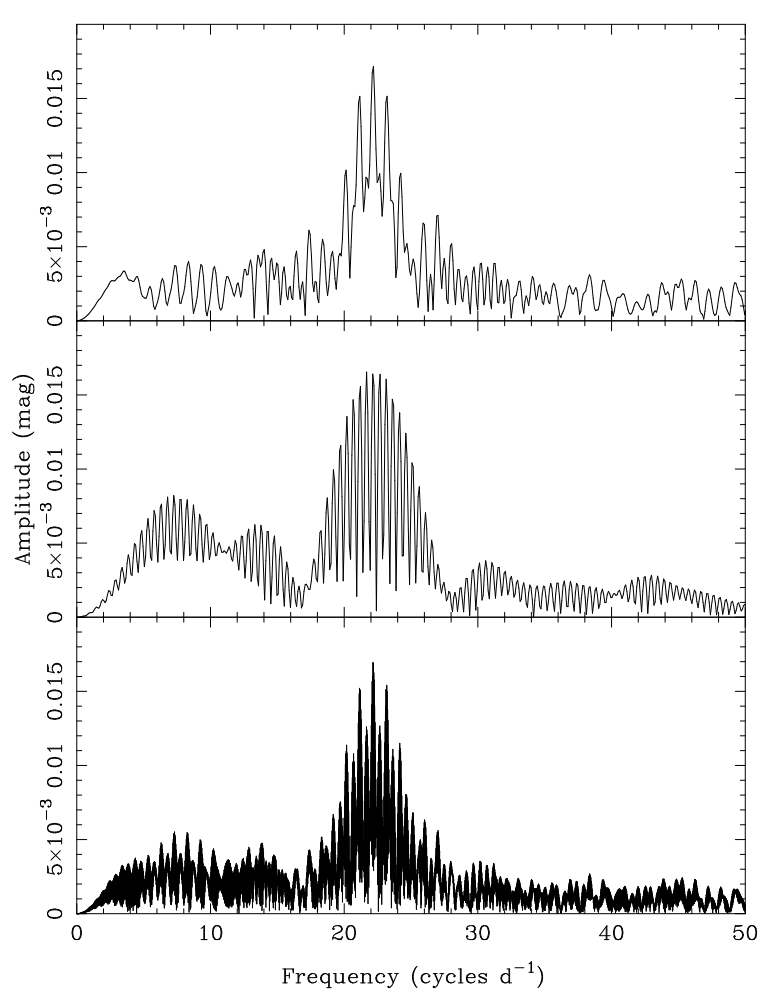

Figure 2. The amplitude spectrum of the all the $0.9 \mathrm{~m}$ data (top panel); all the $2.1 \mathrm{~m}$ data (middle panel); and all the data from both telescopes (bottom panel). The observations from each night were individually detrended by a first order polynomial. In the case of the $2.1 \mathrm{~m}$ observations, fluxes from the four channels have been co-added.

trum of all the data; although not visible on the scale of Figure 2, the main peak is composed of an alias pattern of sub-peaks, spaced 0.031 cycles $\mathrm{d}^{-1}$ apart. The frequencies for the peaks with the largest amplitudes are listed in Table 2. A nonlinear least squares fitting procedure, with a starting guess for the frequency corresponding to the largest-amplitude alias gave $f_{\max }=22.178 \pm 0.00016$ cycles day $^{-1}[P=0.0450897 \pm(3 \times 10-7)$ days $]$ and amplitude $=17.2 \pm 0.3 \mathrm{mmag}$. In spite of the small formal frequency error, we would hesitate to choose the correct alias based on 
Table 3. Amplitudes and phases (with respect to the first observation) of the variations in the different wavebands. The contents of this Table were derived by fitting a sinusoid with $f=22.178$ c/d to the $U, B, V$ and $R$ data by linear least squares. The formal standard errors are given in brackets below each estimate.

\begin{tabular}{ccccc}
\hline & $U$ & $B$ & $V$ & $R$ \\
\hline $\begin{array}{c}\text { Amplitude (mmag) } \\
\text { (s.e.) }\end{array}$ & 19.4 & 17.3 & 14.8 & 11.8 \\
& $(0.3)$ & $(0.2)$ & $(0.3)$ & $(0.3)$ \\
Phase & 1.64 & 1.61 & 1.65 & 1.51 \\
(s.e.) & $(0.02)$ & $(0.01)$ & $(0.02)$ & $(0.03)$ \\
\hline \hline
\end{tabular}

the photometry alone. Some of the reasons for our misgivings are: the two large peaks in the amplitude spectrum have almost identical heights; corrections for drifts in the photometry zeropoints are uncertain; and the $2.1 \mathrm{~m}$ data used in the procedures described above was the simple sum of the fluxes in all four filters. This choice was based on the desire to maximise the available flux, but implies a distinct mismatch of the wavebands of the two sets of observations. It is quite conceivable that the relative heights of the amplitude spectrum aliases might change if a different combination of Stiening photometer fluxes is used. However, we have additional constraints imposed by spectroscopic observations. We show below that the orbital period of KPD $0422+5421$ is $P=0.0901795 \pm\left(3 \times 10^{-7}\right)$ days, which corresponds to a period of $P=2 / f_{\max }$.

A linear least squares programme was used to fit the frequency as determined above to the combined two $2.1 \mathrm{~m}$ runs; this was done separately for each of the four Stiening photometer wavebands. The amplitudes are given in Table 3. Although formal standard errors of the fits are given, these should be viewed only as very rough guides. In particular, errors in different waveband datasets are probably highly correlated, so that the evaluation of e.g. colour phase differences on the basis of the formal errors may be misleading.

\section{SPECTROSCOPIC OBSERVATIONS AND ANALYSIS}

We obtained 14 spectra of KPD $0422+5421$ starting at HJD $2,450,756.8586$ (1997 November $4.3583 \mathrm{UT}$ ) with the $2.7 \mathrm{~m}$ telescope at the McDonald Observatory using the Large Cass Spectrograph, a $600 \ell / \mathrm{mm}$ grating (blazed at $4200 \AA$ ), and the TI1 $800 \times 800 \mathrm{CCD}$. The resulting spectral resolution is $\approx 3.5 \AA$ (FWHM) with wavelength coverage of 3525 $4940 \AA$. The exposure times ranged from 10 to 20 minutes, and the signal-to-noise ratios in the final reduced spectra were $\approx 20-40$ in the continuum near $\mathrm{H} \beta$. There were cirrus clouds present, so the spectra could not be placed on an absolute flux scale. Instead, we used nine observations of five different spectrophotometric flux standards from the nights of November 1-3, 1997 (which were photometric) to calibrate out the instrumental response. Since the slit could not be rotated to follow the parallactic angle, most of our program objects were observed at hour angles near zero. The relative flux scale is reasonably accurate between $\mathrm{H} \beta$ and the Balmer jump-however the relative calibration at
Table 4. The orbital parameters for KPD $0422+5421$.

\begin{tabular}{cc}
\hline Parameter & Value \\
\hline spectroscopic period (days) & $0.0907 \pm 0.0020$ \\
$2 \times$ photometric period (days) & $0.0901795 \pm\left(3 \times 10^{-7}\right)$ \\
$T_{0}$ (spect) (HJD 2,450,000+) & $756.9381 \pm 0.0010$ \\
$T_{0}$ (photo) (HJD 2,450,000+) & $785.8199 \pm 0.0050$ \\
$K_{\text {sdB velocity }\left(\mathrm{km} \mathrm{s}^{-1}\right)}$ & $237 \pm 18$ \\
$\gamma$ velocity $\left(\mathrm{km} \mathrm{s}^{-1}\right)$ & $-57 \pm 12$ \\
$f(M)\left(M_{\odot}\right)$ & $0.126 \pm 0.029$ \\
\hline \hline
\end{tabular}

either end of the covered spectral range is suspect in part because some of the KPD $0422+5421$ spectra were obtained at relatively large airmass (1.5-2.0).

We used the cross-correlation technique of Tonry \& Davis (1979) to determine the radial velocities of the KPD $0422+5421$ spectra. The cross-correlation functions (CCFs) were computed over the wavelength interval 3800-4920. We used a synthetic spectrum generated from a model atmosphere (see below) as the template spectrum (although our results were almost identical when the first observation was used as the template). In each case the CCFs were strong and well-defined. The velocities corresponding to the centroid of the CCF peaks were determined by a parabolic fit to the six pixels surrounding the maximum.

Large radial velocity variations were evident. We fitted a four-parameter sinusoid to the 14 radial velocities. The best-fitting sinusoid had $\chi_{\nu}^{2}=6.5$, an indication that the errors on the radial velocities were probably too small. We scaled the errors on the individual velocities to give $\chi_{\nu}^{2}=1$ for the fit. The resulting spectroscopic elements are given in Table 4 . Note that the spectroscopic period of $P_{\text {spect }}=$ $0.0907 \pm 0.0020$ days is roughly twice the photometric period derived above. The spectroscopic period and the velocity semi-amplitude of $K_{\mathrm{sdB}}=237 \pm 18 \mathrm{~km} \mathrm{~s}^{-1}$ imply a mass function of the (unseen) companion star of

$f(M)=\frac{P K_{\mathrm{sdB}}^{3}}{2 \pi G}=\frac{M_{\mathrm{comp}}^{3} \sin ^{3} i}{\left(M_{\mathrm{comp}}+M_{\mathrm{sdB}}\right)^{2}}=0.126 \pm 0.029 M_{\odot}(1)$

where $M_{\text {comp }}$ is the mass of the unseen companion star, $M_{\mathrm{sdB}}$ is the mass of the sdB star, and $i$ is the orbital inclination. The above equation implies $M_{\text {comp }}>0.126 \pm 0.029 M_{\odot}$.

The fact that the photometric period is half the spectroscopic period suggests that the optical modulations we observe are due to the well-known "ellipsoidal" variations. The cause of the ellipsoidal modulations is easy to understand. The sdB star we observe is slightly distorted by the unseen companion star. As the sdB star moves around in its orbit, its projected area on the sky changes. The changes in the projected area give rise to the observed changes in the total flux. We expect to observe the maximum flux at the quadrature phases when the sdB star is viewed "side-on." Similarly, we will see minima in the light curve during the conjunction phases when the sdB star is viewed "end-on." The photometric minimum at the spectroscopic phase 0.25 (the superior conjunction of the sdB star) will be slightly deeper due to gravity darkening (see Avni 1978 and the discussion below). Thus we can use the spectroscopically determined phase to select the correct alias period from among the several of the most likely candidates. We find 


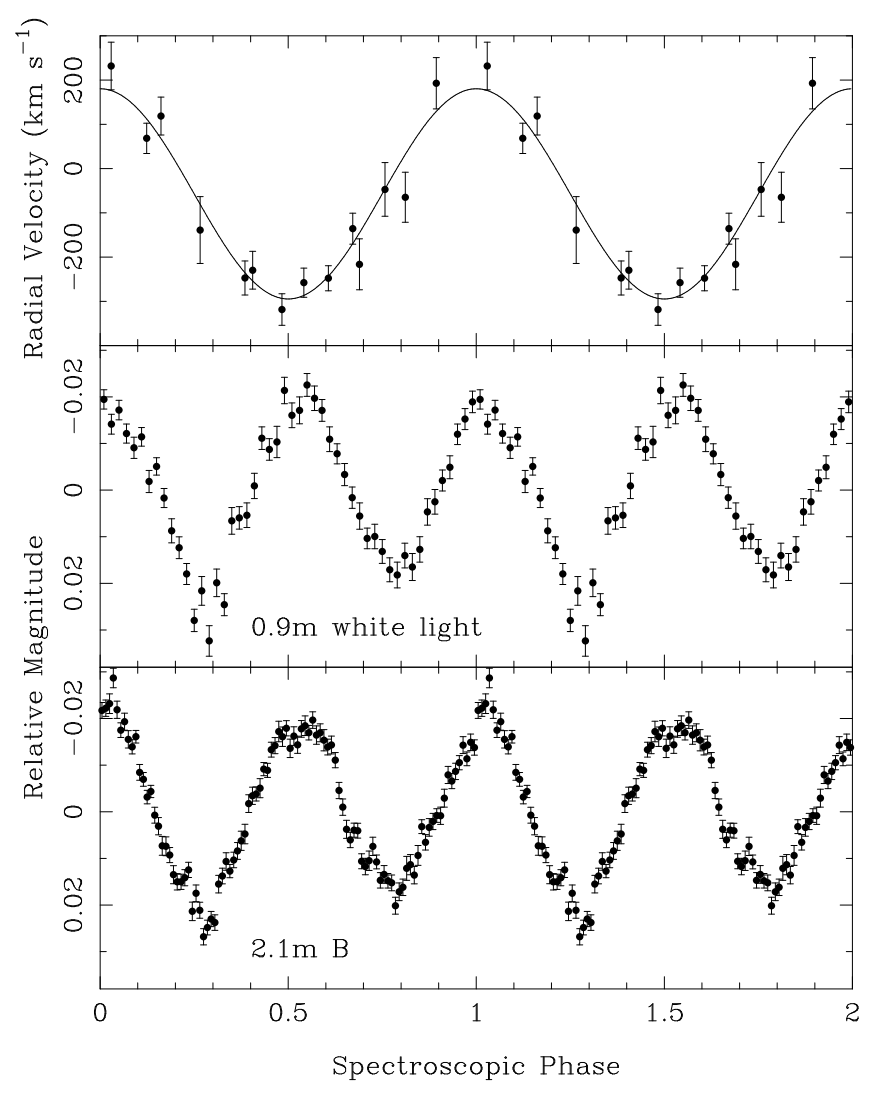

Figure 3. Top: The folded radial velocities of KPD $0422+5421$ and the best-fitting sinusoid. Middle: The folded light curve from the $0.9 \mathrm{~m}$ (white light), binned into 50 phase bins. Bottom: The folded light curve from the $2.1 \mathrm{~m}$ ( $B$ band), binned into 100 phase bins. The errors shown for the binned light curves are the errors of the mean in each bin.

that only the period of $2 / f_{\max }=0.0901795 \pm\left(3 \times 10^{-7}\right)$ days correctly phases the 0.9 and 2.1 meter photometric data with respect to the radial velocity curve. The time of the superior conjunction of the sdB star, as determined from the $2.1 \mathrm{~m}$ data, is $T_{0}$ (photo) $=$ HJD 2, 450, $785.8199 \pm 0.005$. The values of $T_{0}$ (photo) and $T_{0}$ (spect) differ in phase by $\Delta \phi=320.270 \pm 0.013$, consistent with the expected value of $\Delta \phi=320.25$. The folded radial velocity curve and the folded light curves from the $0.9 \mathrm{~m}$ (white light) and the $2.1 \mathrm{~m}$ $(B)$ are shown in Figure 3. Models of the ellipsoidal light curve will be discussed in Section 1 below.

We created a "restframe" spectrum of KPD 0422+5421 by Doppler correcting all 14 spectra to zero velocity and averaging them. This restframe spectrum is displayed in Figure 4. Two important points should be noted. First, the spectrum does not show any obvious lines from the companion, quite unlike typical "composite" spectrum subdwarf stars from the Palomar-Green (PG) survey (Ferguson, Green, \& Liebert 1984; Green, Schmidt, \& Liebert 1986; Orosz, Wade, \& Harlow 1997). Second, the He lines are relatively weak. We will discuss models of the spectrum in Section 5 below.

\section{CONSTRAINTS ON THE SYSTEM GEOMETRY FROM LIGHT CURVE MODELS}

We analyzed the $U$ and $B$ light curves from the observations on the $2.1 \mathrm{~m}$ telescope, by using the Wilson-Devinney (W-D) code (Wilson \& Devinney 1971; Wilson 1979). Only the $U$ and $B$ light curves were used in the modelling process as these were less noisy than the $V$ and $R$ data. This may be due in part to the greater difficulty in correcting the observations through the two red filters for random atmospheric transparancy changes.

We can draw some basic conclusions about the system even before we begin the modelling. The mass of the sdB star is probably not very different from $0.5 M_{\odot}$ (Saffer et al. 1994). We can compute the orbital separation as a function of the total system mass from Kepler's third law:

$a^{3}=\frac{P^{2} G M_{\text {total }}}{4 \pi^{2}}$.

We find for KPD $0422+5421$ that

$\frac{a}{R_{\odot}}=0.846\left(\frac{M_{\text {total }}}{M_{\odot}}\right)^{1 / 3}$.

For mass ratios near 1, the radius of the companion's Roche lobe is approximately $38 \%$ of the orbital separation (Eggleton 1983$)$, which for a total system mass near $1 M_{\odot}$, is about $0.35 R_{\odot}$ for $\mathrm{KPD} 0422+5421$. If the companion star were on the main sequence, then it would be later type than about M2 in order to have a radius small enough to fit inside the Roche lobe (Gray 1992). Even then, it would still fill a sizable fraction of its Roche lobe. Hence we would expect to see a reflection effect where the irradiation of the $M$ star by the hot sdB star causes extra light to be observed near the spectroscopic phase 0.75 , as in the case of HW Vir (Wood et al. 1993). It is clear from Figure 3 that the light curves are ellipsoidal - there is no hint of a reflection effect. This rules out the possibility that the companion is a cool mainsequence star. The mass function rules out a brown dwarf of smaller radius. In principle, there would not be any sizable reflection effect if the two stars had nearly the same temperature. However, if the companion star had a radius and temperature similar to the sdB star, we would observe a double-lined spectroscopic binary since $L_{\mathrm{comp}} \approx L_{\mathrm{sdB}}$. The system is single-lined, however. We conclude that the companion star is much smaller than the sdB star, and therefore probably is a white dwarf.

The light curves were fit using "mode 2" of the W-D code where "star 1 " was the sdB star. Mode 2 is the normal mode used when neither star is in contact with its Roche lobe. We assumed circular orbits and synchronous rotation, both reasonable assumptions considering the proximity of the two stars. The monochromatic fluxes were computed assuming blackbodies. We fixed the polar temperature of the sdB star at $T_{1}=26000 \mathrm{~K}$, and assigned limb darkening coefficients of $u_{1}=0.31$ and 0.29 for the $U$ and $B$ bands, respectively (Wade \& Rucinski 1985). The sdB star has a radiative envelope, so its gravity darkening exponent $g_{1}$ was set to 1 and its bolometric albedo $A_{1}$ for reflective heating and re-radiation was set to 1 . Since the white dwarf contributes less than $0.1 \%$ of the total flux, the values of $T_{2}$, $u_{2}, g_{2}$, and $A_{2}$ are relatively unimportant. Thus we allowed 


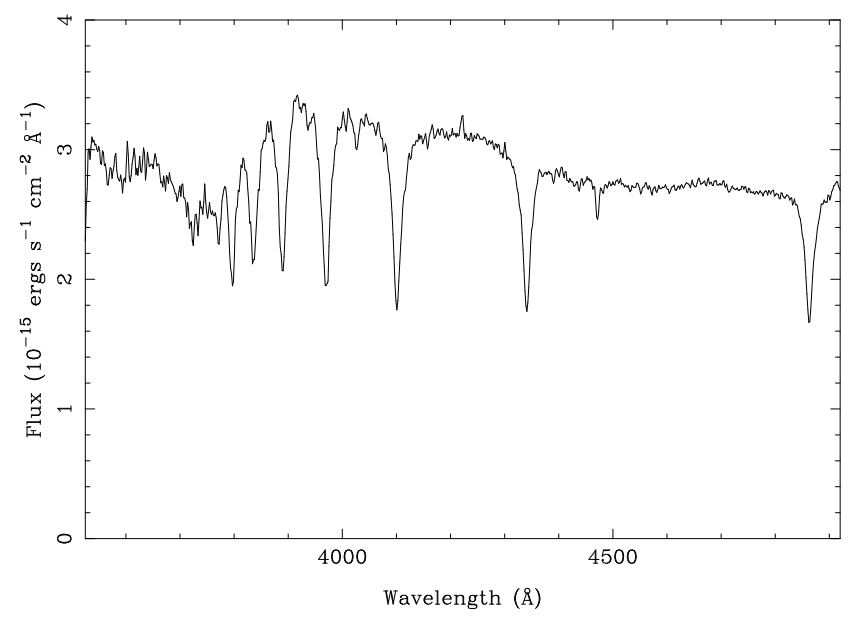

Figure 4. The rest frame spectrum of KPD $0422+5421$, which is the average of the 14 spectra Doppler corrected to zero velocity. The signal-to-noise ratio is about 130 near $\mathrm{H} \beta$.

$T_{2}$ to be a free parameter and set $u_{2}=u_{1}, g_{2}=g_{1}$, and $A_{2}=A_{1}$. The free parameters in the model fits are the inclination $i$, the mass ratio $q=M_{\text {comp }} / M_{\mathrm{sdB}}, T_{2}$, and the surface potentials $\Omega_{1}$ and $\Omega_{2}$. The effective wavelengths of the Stiening $U$ and $B$ filters were taken to be $3460 \AA$ and $4370 \AA$, respectively (Wood et al. 1993).

We used a variation of the "gridls" program given in Bevington (1969) to optimize the parameter values. We defined the optimal light curve solution to be the one which minimized the $\chi^{2}$ fit to both the normalized $U$ and $B$ curves (in magnitude units) simultaneously. We also used the "differential corrections" routine of the W-D code to check our results. We are confident we have found the global $\chi^{2}$ minimum (rather than a local minimum) since we started the optimization routine from a large number of widely separated regions in parameter space. We show in Figure 5 the best fit solution to the $U$ and $B$ light curves and the residuals. The overall fit is reasonably good with $\chi_{\min }^{2}=364.36$ for 200 data points. The residuals in the $U$ and $B$ filters have standard deviations of 0.0029 and 0.0025 magnitudes, respectively. Table 5 summarizes the assumed input parameters and the derived parameters from the model light curve fits.

The statistical errors of the parameters derived from the light curve models were derived in the following way. The parameter of interest (call it $a_{i}$ ) was slightly altered from its optimal value $\left(a_{i}^{1}=a_{i}^{0}+\delta\right)$ and held fixed while the other free parameters were adjusted to give the minimum $\chi^{2}$. The process was repeated $\left(a_{i}^{2}=a_{i}^{1}+\delta\right.$, etc. $)$ until $\chi^{2}$ differed substantially from $\chi_{\min }^{2}$. Figure 6 shows the $\chi^{2}$ vs. $i$ and the $\chi^{2}$ vs. $q$ curves. A change in the inclination $i$ of $\approx 0.5^{\circ}$ is required to force $\chi^{2}$ to change by 1 . We adopt $\sigma_{i}=0.50^{\circ}$, although we note that this is a rough approximation since the curve is not parabolic and $\chi_{\min }^{2}$ is much larger than the number of data points fit. Based on the $\chi^{2}$ vs. $q$ curve, we adopt $\sigma_{q}=0.15$ for the sake of discussion (and with the same caveats discussed for $\sigma_{i}$ ). The quoted errors on the other parameters given in Table 5 were derived assuming Gaussian errors. Eggleton's (1983) formula was used to compute the

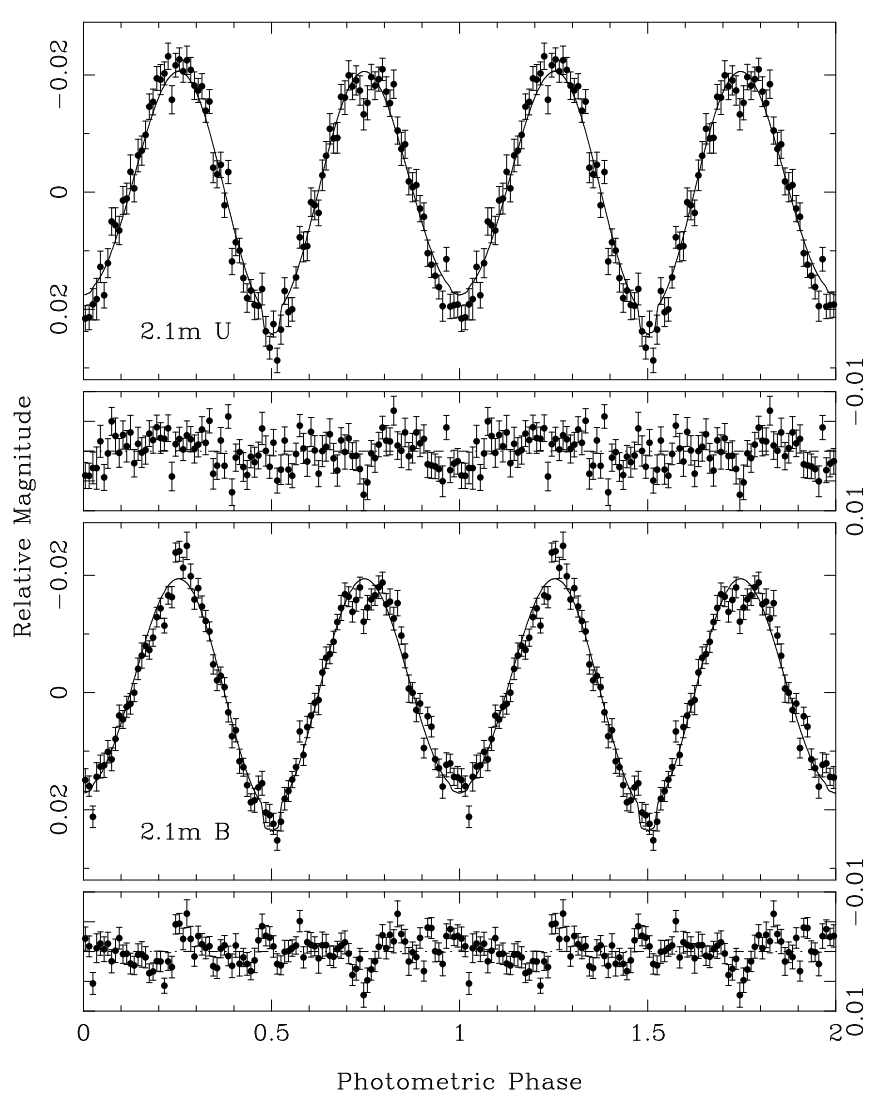

Figure 5. From top to bottom: The $U$ light curve and the best fit model; the $U$ residuals in the sense of data minus model; the $B$ light curve and fit; and the $B$ residuals. Each point has been plotted twice. See Table 5 for the light curve model parameters.

effective radii of the Roche lobes

$$
\begin{aligned}
\frac{R_{R l}(\mathrm{sdB})}{a} & =\frac{0.49 q^{-2 / 3}}{0.6 q^{-2 / 3}+\ln \left(1+q^{-1 / 3}\right)} \\
\frac{R_{R l}(\mathrm{comp})}{a} & =\frac{0.49 q^{2 / 3}}{0.6 q^{2 / 3}+\ln \left(1+q^{1 / 3}\right)} .
\end{aligned}
$$

The W-D code computes the radii of the components using the values of $\Omega_{1}, \Omega_{2}$, and the orbital separation $a$. The sdB star is well within its Roche lobe.

We tried model fits with the temperature of the sdB star set to $25,000 \mathrm{~K}$ and to $27,000 \mathrm{~K}$. The best-fit values of $i$ and $q$ were nearly identical to those values given in Table 5 . In that same vein, we also tried model fits where the limb darkening coefficients were set to 0.25 and 0.35 for both filters. Again, the best-fit values of $i$ and $q$ did not change significantly. Finally, we found that the best-fit values of $i$ and $q$ did not change significantly when the effective wavelengths of the $U$ and $B$ filters were changed by $100 \AA$. We conclude that our results presented in Table 5 are insensitive to the exact values chosen for the sdB parameters.

We emphasize that we have only estimated the statistical errors and not any systematic errors. There are no doubt systematic errors present, given the small light curve amplitudes and the nature of the observations (i.e. high speed 


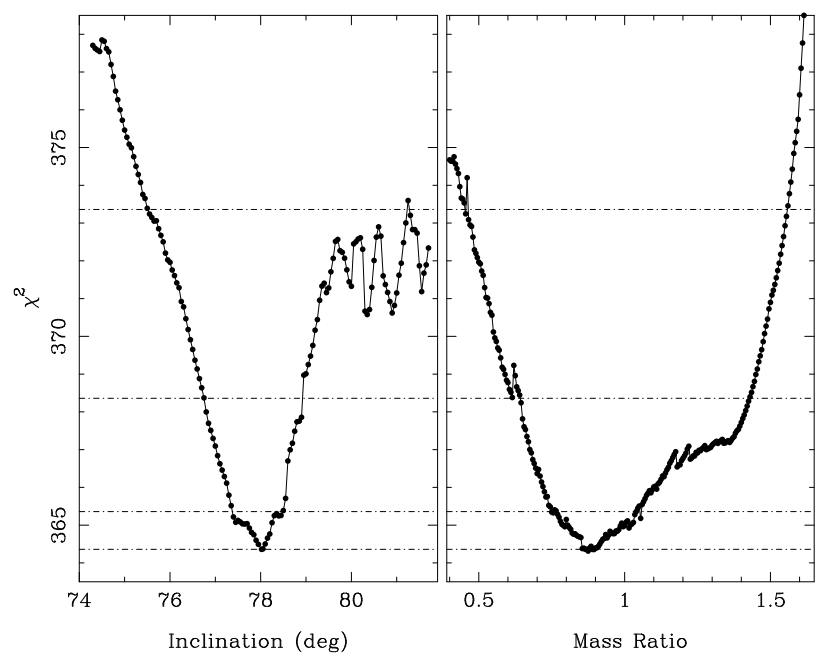

Figure 6. Left: The $\chi^{2}$ vs. $i$ curve. The value of $i$ was fixed at each value along the curve and the other parameters were adjusted as to give the lowest $\chi^{2}$. The dashed-dotted lines denote $\chi_{\min }$, $\chi_{\min }+1, \chi_{\min }+4$, and $\chi_{\min }+9$. Right: The $\chi^{2}$ vs. $q$ curve.

Table 5. Fitted parameters for KPD $0422+5421$.

\begin{tabular}{cc}
\hline Parameter & Value \\
\hline$i(\mathrm{deg})$ & $78.02 \pm 0.50$ \\
$q$ & $0.87 \pm 0.15$ \\
$T_{2}(\mathrm{~K})$ & $\approx 4040$ \\
$\Omega_{1}$ & $\approx 5642 \pm 0.24$ \\
$\Omega_{2}$ & \\
& $0.72 \pm 0.6086$ \\
$M_{\mathrm{sdB}}\left(M_{\odot}\right)$ & $0.62 \pm 0.18$ \\
$M_{\mathrm{comp}}\left(M_{\odot}\right)$ & $0.248 \pm 0.008$ \\
$R_{\mathrm{sdB}}\left(R_{\odot}\right)$ & $\approx 0.01$ \\
$R_{\mathrm{comp}}\left(R_{\odot}\right)$ & $5.50 \pm 0.21$ \\
$\log g_{\mathrm{sdB}}(\mathrm{cgs})$ & $\approx 7.9$ \\
$\log g_{\mathrm{comp}}(\mathrm{cgs})$ & $0.36 \pm 0.05$ \\
$R_{R l}(\mathrm{sdB})\left(R_{\odot}\right)$ & $0.35 \pm 0.03$ \\
$R_{R l}(\operatorname{comp})\left(R_{\odot}\right)$ & $137 \pm 4$ \\
$a\left(R_{\odot}\right)$ & $0.93 \pm 0.11$ \\
\hline
\end{tabular}

photometry). However, the white light curve from the $0.9 \mathrm{~m}$ and the $B$ light curve from the $2.1 \mathrm{~m}$ are very similar in amplitude, and their relative phases are as expected. There is also nothing unusual about the derived component masses. The mass of the sdB star $\left(0.72 \pm 0.26 M_{\odot}\right)$ is consistent with the "canonical" extended horizontal branch mass of $0.5 M_{\odot}$ (Caloi 1989; Dorman, Rood, \& O'Connell 1993; Saffer et al. 1994). Webbink (1990, see also Warner 1995) finds a mean white dwarf mass of $0.74 \pm 0.04 M_{\odot}$ in 84 cataclysmic variable binaries, and the mean mass of white dwarfs in the field is near 0.56 $M_{\odot}$ (Bergeron, Saffer, \& Liebert 1992). The mass of the white dwarf in KPD $0422+5421\left(0.62 \pm 0.18 M_{\odot}\right)$ is consistent with either of these. These considerations suggest that the systematic errors are reasonably small and that ou derived parameters are trustworthy.

A close examination of the model light curve near the photometric phase 0.5 (when the sdB star is behind the com- panion) shows that the white dwarf companion passes in front of the $\mathrm{sdB}$ star, causing a dip of $\approx 0.005$ magnitudes which lasts $\approx 0.05$ in phase (6.5 minutes). There is also a very slight distortion at phase 1.0 where the nearly invisible white dwarf is totally eclipsed by the sdB star. Interestingly enough, the white-light curve from the $0.9 \mathrm{~m}$ shows a possible indication of a small dip near the spectroscopic phase 0.25 (i.e. the photometric phase 0.5). Caution suggests that the observation of a transit be considered only tentative. Since the depth of the depression caused by the transit is only $\approx 0.005$ magnitudes, its exact shape is quite sensitive to slight drifts in the transparency, etc. Extended observations with a CCD on a large telescope (where one can use reasonably short exposures) should be done to confirm the existence of the transit. The use of a CCD enables one to do precise differential photometry over many orbital cycles.

\section{SUBDWARF ATMOSPHERIC PARAMETERS}

The effective temperature $T_{\text {eff }}$ and surface gravity $\log g$ of the subdwarf were determined using a grid of synthetic spectra. LTE hydrogen and helium line-blanketed model atmospheres were constructed using version 178 of the atmosphere code TLUSTY (Hubeny 1988), for solar abundances and for reduced helium abundance, $[\mathrm{He} / \mathrm{H}]=-0.5,-1.0$, and -1.5 (where square brackets denote the logarithmic number ratio relative to solar). Detailed spectra were synthesized on the interval $3780-4910 \AA$ using version 41 of SYNSPEC (Hubeny, Lanz \& Jeffery 1994); H and He I lines were computed using line broadening tables, while lines from other elements were computed using Voigt profiles and data from the Kurucz atomic line list. A microturbulent velocity parameter of $4 \mathrm{~km} \mathrm{sec}^{-1}$ was assumed. An approximate NLTE treatment of line opacity based on second-order escape probability was used in the spectrum synthesis (see Hubeny, Harmanec \& Stefl 1986). The spectra were then convolved and sampled to match the dispersion (1.77 $\AA$ pixel $^{-1}$ ) and instrumental resolution $(3.5 \AA)$. On the assumption that the subdwarf's rotation is tidally synchronized to the binary orbital period, we included a rotational broadening of $V_{\text {rot }} \sin i=150 \mathrm{~km} \mathrm{sec}^{-1}$, which is less than the FWHM of the instrumental profile. At each value of $[\mathrm{He} / \mathrm{H}]$, models and spectra were computed at spacings of $1000 \mathrm{~K}$ for $T_{\text {eff }}=23000-28000 \mathrm{~K}$, and spacings of 0.2 for $\log g=5.0-5.8$. For $[\mathrm{He} / \mathrm{H}]<0$, the grid resolution was increased in the neighborhood of the best fit, to $500 \mathrm{~K}$ for $T_{\text {eff }}=24000-27000 \mathrm{~K}$ and 0.1 for $\log g=5.2-5.6$.

The models and the restframe spectrum were normalized to their continua, and the $\chi^{2}$ and rms residuals were computed using most of the spectrum between $3900 \AA$ and $4900 \AA$. The $\mathrm{H} \beta$ profile and nearby continuum were not well fit by the models, in part due to the relatively poor calibration near the ends. The CII line at $4266.7 \AA$ is much weaker in the data than in the model spectra. After some experimentation, final fitting regions were chosen to be $3949.0 \leq \lambda \leq 4210.0,4282.0 \leq \lambda \leq 4394.0$, and $4460.0 \leq \lambda \leq 4486.0$. This excludes the CII line, some bad pixels near $4222 \AA$, and the region near $4430 \AA$ which has a weak interstellar absorption band. The best-fitting model (Figure 7 has $T_{\text {eff }}=25,000 \mathrm{~K}, \log g=5.4$, and $[\mathrm{He} / \mathrm{H}]=$ 


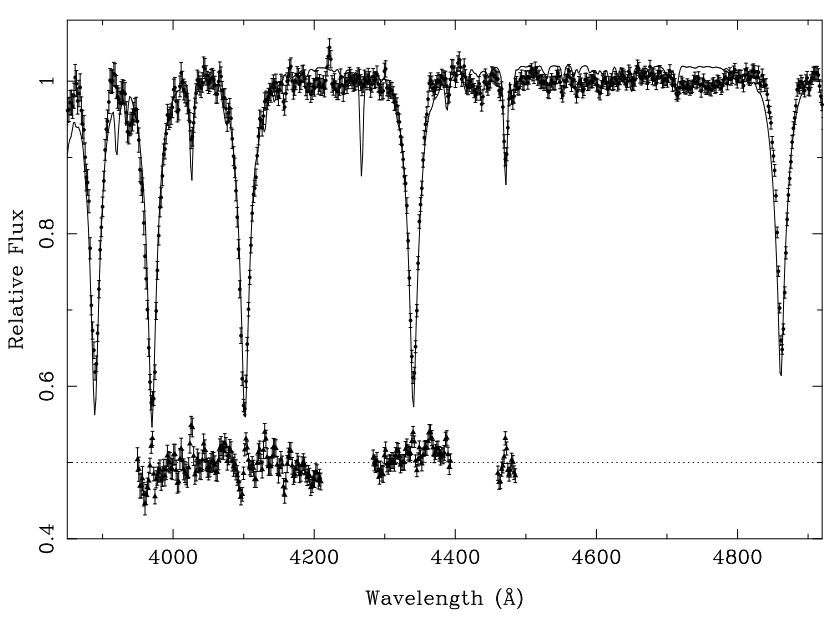

Figure 7. The restframe spectrum (filled circles) is displayed with the best-fitting model (solid line) and the residuals in the sense of data minus model (filled triangles), offset by 0.5 units upward. The CII line at $4266.7 \AA$ was not included in the fitting region.

-1.0. The rms residual of the fit is 0.018 (unit $=$ normalized continuum). For comparison, the value of $\log g$ derived from the photometrically determined mass and radius of the sdB star is $\log g=5.50 \pm 0.21$ (Table 5). Keeping $T_{\text {eff }}, \log g$, and $[\mathrm{He} / \mathrm{H}]$ at their best values, we find that an approximate match of the models to the strength of the CII line at $4266.7 \AA$ requires a reduction in the carbon abundance by a factor of ten or more with respect to solar. Our resolution and signal-to-noise ratio are insufficient to make further deductions about abundances of metals.

The estimated sizes of the $1 \sigma$ statistical errors are $\sigma_{T} \approx 1500 \mathrm{~K}$ and $\sigma_{\log g} \approx 0.1$. There is a positive correlation between the estimated parameters $T_{\text {eff }}$ and $\log g$, in the sense that forcing a displacement of 0.1 dex in $\log g$ requires a displacement of $\sim 700 \mathrm{~K}$ to minimize $\chi^{2}$. Forcing $[\mathrm{He} / \mathrm{H}]$ to a value away from the best fitting -1.0 pushes $T_{\text {eff }}$ and $\log g$ along this correlation. Our values of $T_{\text {eff }}=25,000 \pm 1500 \mathrm{~K}$ and $\log g=5.4 \pm 0.1$ are consistent with those values communicated by Saffer $\left(T_{\text {eff }}=26,050, \log g=5.51,[\mathrm{He} / \mathrm{H}]\right.$ unknown). If we had used SYNSPEC to generate synthetic spectra using line-blanketed models from Kurucz (1994 CDROM distribution), we estimate that our inferred $T_{\text {eff }}$ would be $\sim 1500 \mathrm{~K}$ lower with no change in $\log g$, based on comparisons made at $[\mathrm{He} / \mathrm{H}]=0$. We stress that we have not calibrated our parameter fitting procedure directly against stars analysed by other workers.

The spectroscopic value of $\log g$ can be used to make an independent estimate of the radius of the sdB star. As a function of the total mass the radius of the $\mathrm{sdB}$ star is

$\frac{R_{\mathrm{sdB}}}{R_{\odot}}=\left[\left(\frac{M_{\text {total }}}{M_{\odot}}\right)\left(\frac{g \odot}{g}\right)\left(\frac{1}{1+q}\right)\right]^{1 / 2}$

where $q \equiv M_{\text {comp }} / M_{\text {sdB }}$ and $M_{\text {total }} \equiv M_{\text {sdB }}+M_{\text {comp }}$. On the other hand, the radius of the $\mathrm{sdB}$ star is given by the light curve solution as a fraction $\mathcal{F}$ of the effective Roche lobe radius $R_{\mathrm{Rl}}(q)$. Thus using Kepler's third law,

$R_{\mathrm{sdB}}=\mathcal{F} R_{\mathrm{Rl}}(q)\left[\frac{P^{2} G M_{\text {total }}}{4 \pi^{2}}\right]^{1 / 3}$

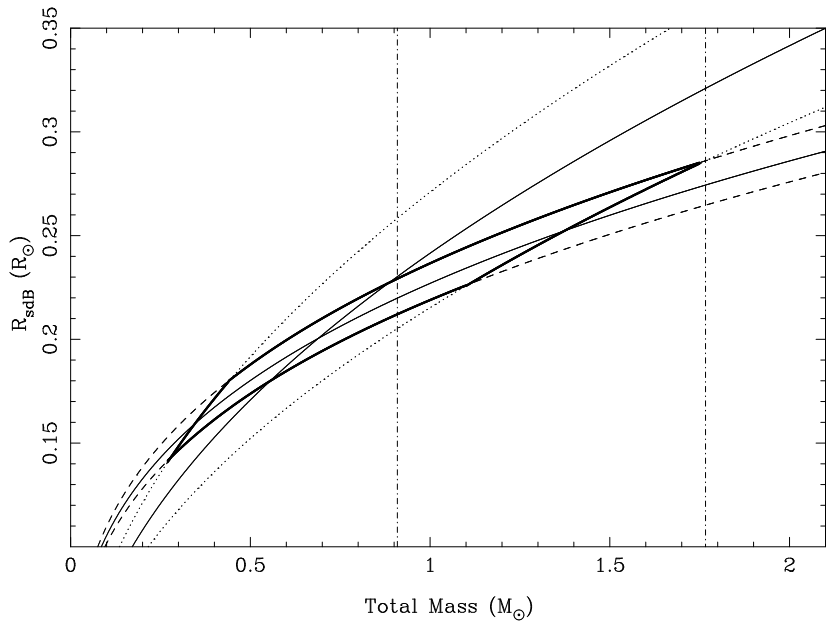

Figure 8. The relations given in Equations 1 and 6 are plotted as the solid lines. The dotted lines show Equation 5 using the $1 \sigma$ errors on $\log g$. The dashed lines show Equation 6 using the $1 \sigma$ errors on $q$ The region where the two $1 \sigma$ regions overlap is outlined with the thick line. Finally, the $1 \sigma$ range of $M_{\text {total }}$ allowed by the mass function is shown with the vertical dashed-dotted lines.

where $R_{\mathrm{Rl}}(q)$ is given in Equation 4 . In Figure 8 we plot equation 1 using $\log g=5.4$ and Equation 6 using $q=0.87$. The two curves cross at $M_{\text {total }} \approx 0.7 M_{\odot}$. We also show the $1 \sigma$ limits on each curve $\left(\sigma_{\log g}=0.1\right.$ and $\left.\sigma_{q}=0.15\right)$. The region where the two error bands overlap is outlined with the thick lines. An extreme range of $0.25 \leq M_{\text {total }} \leq 1.75 M_{\odot}$ is allowed, compared with the $1 \sigma$ range of $0.91 \leq M_{\text {total }} \leq$ $1.77 M_{\odot}$ allowed by the mass function and the photometrically determined values of $q$ and $i$. We conclude that the radius derived from our spectroscopic value of $\log g$ is basically compatible with the radius derived from the photometric determination of $q$ and $i$, while favoring lower values of $M_{\text {total }}$.

A measurement of the projected rotational velocity of the $\operatorname{sdB} \operatorname{star} V_{\text {rot }} \sin i$ would provide another measurement of its radius:

$R_{\mathrm{sdB}}=V_{\mathrm{rot}} \sin i\left(\frac{P}{2 \pi \sin i}\right)$,

provided the sdB star is synchronously rotating. Our present spectral resolution is not sufficient to measure $V_{\text {rot }} \sin i$ for KPD 0422+5421 directly. Such a measurement would provide another useful constraint on the total system mass through the relations given in Equations 8 and 6 .

\section{THE REDDENING FROM ABSOLUTE PHOTOMETRY AND THE DISTANCE}

Absolute photometry of KPD $0422+5421$ in the Johnson $U B V$ and Strömgren systems was obtained by Downes (1986) and Wesemael et al. (1992) respectively. The results are: $V=14.66, B-V=+0.20, U-B=-0.65$ (no errors are quoted); and $y=14.682 \pm 0.018, b-y=0.141 \pm 0.011$, $u-b=0.477 \pm 0.001, m_{1}=0.063 \pm 0.019$. Since the photometric variations are quite small and in phase at different wavelengths, it may be assumed that the accuracy of the absolute photometry is largely unaffected by the cyclical variations. 
Koen et al.

Comparison of the $U-B$ and $B-V$ indices with those of a large collection of hot subdwarfs given in Fig. 2 of Kilkenny et al. (1997b) makes it clear that KPD0422+5421 is anomalously red. In fact, its colours strongly resemble those of hot, subluminous stars with composite spectra, as shown in Fig. 3 of Kilkenny et al. (1997b). However, our spectra do not exhibit any signs of anything but the sdB star. Furthermore, our photometric solution requires the companion to be degenerate. The obvious conclusion is that KPD0422+5421 is substantially reddened.

Further support for this deduction comes from the Strömgren colour indices quoted above. Wesemael et al. (1992) plot reddening lines in a $(u-b, b-y)$ diagram, for a range of assumed sdB temperatures (their Fig. 9); KPD0422+5421 inhabits an area in the diagram with $E(b-$ $y)>0.2$. A crude estimate gives $E(b-y) \approx 0.25$, which is not surprising given that the star is in the galactic plane. By comparing the observed Strömgren photometric indices to stellar atmosphere model predictions, Villeneuve et al. (1995) derive $E(b-y) \approx 0.26$. The value of $E(b-y)=0.25$ implies $E(B-V)=0.36$ (Crawford, Glasky, \& Perry 1970).

To compute the distance to the source, we adopt a temperature of $T_{\text {eff }}=25,000 \pm 1500 \mathrm{~K}$, a radius of $R_{\mathrm{sdB}}=$ $0.249 \pm 0.008 R_{\odot}$, a colour excess of $E(B-V)=0.36 \pm 0.05$, a bolometric correction of $B C=2.5 \pm 0.1$ (Gray 1992), and an apparent $V$ magnitude of $V=14.66$ (Downes 1986). We find $L_{\mathrm{bol}}=21.8 \pm 5.4 L_{\odot}, M_{V}=3.86 \pm 0.29$, and $d=850 \pm 130$ pc. For comparison, Villeneuve, Wesemael, \& Fontaine (1995) find $581 \leq d \leq 1120 \mathrm{pc}$ based on temperatures derived from Strömgren photometry.

\section{DISCUSSION OF SIMILAR SYSTEMS AND EVOLUTIONARY CONSIDERATIONS}

Three issues relating to the binary evolution arise from our study of KPD $0422+5421$.

First, we note that the sdB star, if interpreted as a member of the extreme horizontal branch (EHB), will soon evolve to become the second white dwarf in KPD $0422+5421$. Thus KPD $0422+5421$ is a predecessor to the class of double degenerate binaries. Presumably it has already passed through a common envelope phase. A possible predecessor in turn to KPD $0422+5421$ is a system like HD 185510 (V1379 Aql), which is a 21-day binary containing an evolved compact (sdB) star and a K0 giant (Fekel et al. 1993; Jeffery \& Simon 1997). The mass ratio of HD 185510 is large, $M_{\mathrm{K}} / M_{\mathrm{sdB}} \approx 7.5$ (Fekel et al. 1993), and one may expect that a common envelope stage lies ahead. At that time, HD 185510 may resemble KPD $0422+5421$, with its present sdB star in the rôle of KPD's white dwarf.

Second, what is the present total binary mass? The combined spectroscopic and photometric orbital solution suggests a value tantalizingly close to the Chandrasekhar limit, $M_{\mathrm{Ch}} \approx 1.4 M_{\odot}$, with all that implies for the possibility that KPD $0422+5421$ may become a Type Ia supernova, when the components merge. On the other hand, if the sdB star is truly a core-He burning EHB star, its mass is expected to be close to $0.5 M_{\odot}$, and with $q$ derived from the light curve solution constraining the mass of the unseen white dwarf, the total mass may be considerably less than $M_{\mathrm{Ch}}$. Improved light curves and radial velocity data will help to address this question. In the meantime, it is well to bear in mind the discussion in Jeffery \& Simon (1997) concerning whether the canonical $0.5 M_{\odot}$ for EHB stars is really empirically well-determined.

Third, short-period binary systems of the same type as KPD 0422+5421, namely sdB + white dwarf, are rare, and KPD $0422+5421$ arguably possesses the shortest period of them all. [One double degenerate system has a shorter period: WD 0957-666, $P=0.060993$ days, (Moran, Marsh, \& Bragaglia 1997). The total mass is estimated to be only $0.69 M_{\odot}$, however.] The catalog of Ritter \& Kolb (1998) lists three possible sdB+ "white dwarf" pairs (i.e. sdB stars known to be in close binaries with undetected companions), and the sample of Saffer, Livio, \& Yungelson (1998) contains seven sdB binaries with undetected companions (including two from Ritter \& Kolb's catalog), of which five have known orbital periods. The six objects with known orbital periods (in order of increasing period) are PG $1432+159(P=5.39$ hours), PG 2345+318 ( $P=5.78$ hours), Feige $36(P=8.5$ hours), PG 0101+039 ( $P=13.7$ hours), HZ 22 (=UX CVn, $P=13.77$ hours $)$, and Ton $245(P=2.5$ days $)$. A related sdO + white dwarf(?) system is HD 49798 with $P=1.55$ days (Thackeray 1970). The best known member of the sdB + white dwarf class is HZ 22 (Humason \& Zwicky 1947; Young, Nelson, \& Mielbrecht 1972; Greenstein 1973). Greenstein (1973) and Young \& Wentworth (1982) argue that the unseen companion of HZ 22 is a white dwarf. However, it is also possible that HZ 22 contains a low mass main sequence star companion, so the nature of $\mathrm{HZ} 22$ is still an open question. Another recent sdB+"white dwarf" candidate is V46 in the globular cluster M4 (Kaluzny, Thompson \& Krzeminsky 1997), which is an sdB star that shows an apparently sinusoidal light curve with a period of 1.045 hours. This period is much longer than those seen in the pulsating sdB stars (EC14026 stars, Kilkenny et al. 1997a). However, the nature of the companion star is not known and it is not clear if the photometric period is the orbital period. Since it has the shortest confirmed orbital period, KPD $0422+5421$ will evolve more quickly via gravitational wave radiation of angular momentum than the other sdB + white dwarf systems. Ritter (1986) gives the time required for a detached binary to reach the semi-detached state:

$t_{\mathrm{sd}}=\left(4.73 \times 10^{10} \mathrm{yr}\right) \frac{M_{\text {total }}^{1 / 3}}{M_{\mathrm{comp}} M_{\mathrm{sdB}}} P^{8 / 3}\left[1-\left(\frac{P_{\mathrm{sd}}}{P}\right)^{8 / 3}\right]$

where the masses are in solar units, the periods are in days, and where

$\frac{P_{\mathrm{sd}}}{P}=\left(\frac{R_{\mathrm{sdB}}}{R_{R l}(\mathrm{sdB})}\right)^{3 / 2}$,

which assumes that the radius of the sdB star stays fixed. Using $P_{\mathrm{sd}} / P=0.57 \pm 0.13$, we find that $\log t_{\mathrm{sd}}=8.17 \pm 0.15$ $\left(t_{\mathrm{sd}} \approx 1.5 \times 10^{8}\right.$ years $)$, which is comparable to the core He burning lifetime of an sdB star $\left(\approx 1.5 \times 10^{8}\right.$ years, Dorman, Rood, \& O'Connell 1993). Thus, possibly before the sdB star has evolved to a white dwarf, there will be a further episode of mass exchange. What KPD $0422+5421$ will then look like, and whether it can be identified with any presently known class of objects, takes the subject well beyond the expertise or inclination of the present authors to speculate. 


\section{SUMMARY}

The sdB star KPD $0422+5421$ was found to be a short period detached binary with a period of $P=0.0901795 \pm(3 \times$ $10^{-7}$ ) days. We argue that the companion star is a white dwarf since the companion star is not seen in the spectra, and the synthetic light curve model of the $U$ and $B$ light curves requires its radius to be on the order of $\approx 0.01 R_{\odot}$. We derive component masses of $M_{\mathrm{sdB}}=0.72 \pm 0.26 M_{\odot}$ and $M_{\text {comp }}=0.62 \pm 0.18 M_{\odot} . \mathrm{KPD} 0422+5421$ is one of a small number of known sdB + white dwarf binary systems and represents a poorly observed and short-lived stage of binary star evфlution.

\section{ACKNOWLEDGMENTS}

The authors are grateful to Dr. Rex Saffer for supplying his gravity and temperature estimate of KPD $0422+5421$ before publication. We also thank Peter Eggleton for pointing out to us the intriguing and possibly related system HD 185510. CK thanks Prof. Ed Nather for his hospitality at the University of Texas (Austin); the director of the McDonald Observatory for a generous allotment of telescope time; Rob Robinson and Rae Stiening for the use of the Stiening photometer; and the South African Foundation for Research Development for partially funding some of this work. JO and RW acknowledge partial support from NASA grant NAG 53459 to the Pennsylvania State University. We are grateful to Ivan Hubeny for instruction in the use of his stellar atmosphere codes TLUSTY, SYNSPEC, and ROTINS. This research has made use of the Simbad database, operated at CDS, Strasbourg, France.

\section{REFERENCES}

Avni Y., 1978, in Giacconi R., Ruffini R., Physics and Astrophysics of Neutron Stars and Black Holes, North-Holland, Amsterdam 42

Bergeron P., Saffer R. A., Liebert J., 1992, ApJ, 394, 228

Bevington P. R., 1969, Data Reduction and Error Analysis for the Physical Sciences. McGraw Hill, New York

Caloi V., 1989, A\&A, 221, 27

Crawford D. L., Glasky J. W., Perry C. L., 1970, AJ, 75, 822

Dorman B., Rood R. T., O'Connell R. W., 1993, ApJ, 419, 596

Downes R. A., 1986, ApJS, 61, 569

Eggleton P. P., 1983, ApJ, 268, 368

Fekel F. C., Henry G. W., Busby M. R., Eitter J. J., 1993, AJ, 106,2370

Ferguson D. H., Green R. F., Liebert J., 1984, ApJ, 287, 320

Grauer A. D., Bond H. E., 1981, PASP, 93, 388

Gray D. F., 1992, The Observation and Analysis of Stellar Photospheres. Cambridge University Press, Cambridge

Green R. F., Schmidt M., Liebert J., 1986, ApJS, 61, 305

Greenstein J. L., 1973, A\&A, 23, 1

Horne K., Stiening R. F., 1985, MNRAS, 216, 933

Hubeny I., Harmanec P., Stefl S., 1986, Bull. Astron. Inst. Czechosl. 37, 370

Hubeny I., 1998, Computer Physics Comm. 52, 103

Hubeny I., Lanz T., Jeffery C. S. 1994, in Jeffery C. S., ed., Newletter on Analysis of Astronomical Spectra No. 20, St. Andrews Univ., p. 30

Humason M. L., Zwicky F., 1947, ApJ, 105, 85

Jeffery C. S., Simon T., 1997, MNRAS, 286, 487
Kaluzny J., Thompson I. B., Krzeminsky W., 1997, AJ, 113, 2219

Kilkenny D., Koen C., O'Donoghue D., Stobie R. S., 1997a, MNRAS, 285, 640

Kilkenny D., O'Donoghue D., Koen C., Stobie R. S., Chen A., 1997b, MNRAS, 287, 867

Moran C. K. J., Marsh T. R., Bragaglia A., 1997, MNRAS, 288, 538

Orosz J. A., Wade R. A., Harlow J. J. B., 1997, AJ, 114, 317

Ritter H., 1986, A\&A, 169, 139

Ritter H., Kolb U., 1998, A\&AS, in press

Saffer R. A., Bergeron J., Koester D., Liebert J., 1994, ApJ, 432, 351

Saffer R. A.. Livio M., Yungelson L. R., 1998, ApJ, in press astro$\mathrm{ph} / 9802356$

Thackeray A. D., 1970, MNRAS, 150, 215

Tonry J., Davis M., 1979, AJ, 84, 1511

Villeneuve B., Wesemael F., Fontaine G., 1995, ApJ, 450, 851

Wade R. A., Rucinski S. M., 1985, A\&AS, 60, 665

Warner B., 1995, Cataclysmic Variable Stars. Cambridge University Press, Cambridge

Webbink R. F., 1990, in Mauche C., ed., Accretion Powered Compact Binaries, Cambridge University Press, Cambridge p. 177

Wesemael F., Fontaine G., Bergeron P., Lamontagne R., 1992, AJ, 104, 203

Wilson R. E., Devinney E. J., 1971, ApJ, 166, 605 (W-D)

Wilson R. E., 1992, in Leung K. C., ed., Astronomical Society of the Pacific Conference Series, New Frontiers in Interacting Binary Star Research

Wood J. H., Zhang E. -H., Robinson E. L., 1993, MNRAS, 261, 103

Young A., Nelson B., Mielbrecht R., 1972, ApJ, 174, 27

Young A., Wentworth S. T., 1982, PASP, 94, 815

This paper has been produced using the Royal Astronomical Society/Blackwell Science $\mathrm{AT}_{\mathrm{E} X} \mathrm{X}$ style file. 